\title{
PRODUCTIVIDAD ENERGÉTICA Y FINANCIERA EN FINCAS CAMPESINAS DEL DEPARTAMENTO DE CALDAS. TRES ESTUDIOS DE CASO.
}

\author{
TOBASURA, I. \\ MORENO, F. A. ${ }^{2}$ \\ AYA, $S^{3}$ \\ MORA, J. ${ }^{4}$
}

Manizales, 2011-06-30 (Rev. 2011-10-31)

\section{RESUMEN}

Con el objetivo de estimar la productividad energética y financiera, se estudiaron tres fincas campesinas en los municipios de Filadelfia y Manizales en el departamento de Caldas (Colombia). Las fincas estudiadas se seleccionaron de los clústeres resultantes en la investigación: "Flujo de masas y energía en fincas campesinas de la zona cafetera: vínculos entre la racionalidad campesina y el flujo de materiales"5. Para realizar los análisis, se recabó información con base en entrevistas semiestructuradas aplicadas a cada una de las familias de dichas fincas. Con la información, se elaboraron modelos analógicos, que muestran las entradas y salidas del sistema café, componente principal de la unidad familiar. Se estimó la productividad energética y financiera de cada unidad. Se concluyó que la Finca 1 es la que presenta la mayor eficiencia energética y financiera, debido a su diversidad, reciclaje de materiales y uso de mano de obra familiar. En consecuencia, es la más sustentable.

\section{PALABRAS CLAVE:}

Producción familiar, eficiencia energética, productividad, flujo de materiales.

\section{ENERGETIC AND FINANCIAL PRODUCTIVITY IN PEASANTS' FARMS IN THE DEPARTMENT O F CALDAS. THREE CASE STUDIES}

\begin{abstract}
Three peasants' farms in the municipalities of Filadelfia and Manizales, Caldas (Colombia) were studied with the purpose of estimating energetic and financial productivity. The studied farms were selected from the resulting clusters in the research project "Mass and energy flow in peasants' farms in the coffee triangle: links between peasants' rationality and materials flow" ${ }^{\prime \prime}$. In order to perform the analysis, information was gathered through semi-structured interviews applied to each family from the above mentioned farms. With this information, analogical models were developed which show the coffee system income and expenditure, a main component in family unit. It was concluded that Farm 1 presents the highest energetic and financial efficiency because of its diversity, materials recycling process and use of family work force. Consequently it is the most sustainable one.
\end{abstract}

\section{KEY WORDS:}

Family production, energetic efficiency, productivity, materials flow 


\section{INTRODUCCIÓN}

En los últimos 40 años el desarrollo de la agricultura industrializada, basado en la lógica de la Revolución Verde, ha hecho que las fincas campesinas sean cada vez más especializadas y dependientes de insumos externos, es decir de productos sintéticos de los hidrocarburos. Este hecho ha sido muy significativo en la zona cafetera central del país, donde el monocultivo del café a plena exposición solar constituye el paisaje predominante. Si bien, el resultado trajo consigo una alta productividad y eficiencia económica, con importante generación de divisas y mejoramiento del ingreso familiar, generó problemas sociales y ambientales como exclusión de mano de obra familiar, reducción de la diversidad genética y de la diversificación de los sistemas de producción, contaminación ambiental por residuos sintéticos de difícil degradación o residuos orgánicos contaminantes del suelo y el agua, y pérdida de la energía contenida en los residuos orgánicos no utilizados por el sistema.

No obstante el auge de la agricultura de altos insumos, algunas familias campesinas de la zona cafetera colombiana han configurado, en los últimos años, sistemas de producción diversificados, con mecanismos de reciclaje de los residuos orgánicos, uso de mano de obra familiar, tecnologías locales, menos insumos externos y actividades no agrarias para complementar los ingresos, que les permiten consolidar sistemas de producción con alta capacidad de resiliencia y capacidad de resistencia ante amenazas del entorno ambiental y social.

En estos sistemas diversificados se presenta una amplia producción de biomasa con potencial agrícola o pecuario. Se sabe del uso de materiales fibrosos para producir abonos orgánicos (compost, bochashi) de lenta liberación de nitrógeno; materiales orgánicos de fácil descomposición para el uso inmediato de los nutrimentos; estiércoles que se procesan anaeróbicamente para la producción de gas metano; el uso de materiales de alta relación Carbono/Nitrógeno (C/N) especialmente como enmienda orientada a mejorar la estructura del suelo y productos orgánicos que se asperjan a las plantas (biopreparados). No obstante, las ventajas que ello puede representar para la sostenibilidad de los sistemas de producción y el bienestar de las familias, no todos los productores aprovechan eficientemente la biomasa en sus fincas.

En una investigación realizada por Calderón (2009), en relación con los indicadores de sostenibilidad, se llama la atención sobre el escaso manejo, transformación y reciclaje de residuos sólidos y líquidos en todas las fincas estudiadas; alta dependencia de insumos externos, principalmente, compra de insumos agropecuarios y alimentos en $90 \%$ de las fincas; escasas prácticas agroecológicas para la conservación del suelo en $50 \%$ de las fincas; y escasa producción de alimentos articulados a las cadenas de mercado en $20 \%$ de las fincas estudiadas; en este caso, dos fincas que tienen como único producto para la venta el café, el cual es la base de su economía familiar.

Desde cuando se dieron las primeras alarmas acerca del deterioro de los recursos naturales y el medio ambiente, los académicos y los tomadores de decisiones se han dado a la tarea de encontrar indicadores que permitan estimar la sostenibilidad de los sistemas de producción. No obstante, los esfuerzos realizados con este propósito, aún existe un déficit enorme al respecto. 
Los estudios realizados en esta dirección han partido del conocido "triángulo de la sustentabilidad", que incorpora las dimensiones económica, social y ecosistémica para determinar dicha condición en un agroecosistema en el tiempo. Se trata de estimar, a partir de indicadores en cada dimensión, la capacidad que tienen los agroecosistemas para garantizar en el tiempo la eficiencia económica, la equidad social y la eficiencia energética y diversidad de los ecosistemas.

En la gestión de los agroecosistemas se han encontrado dos lógicas contrastantes con respecto a la función de producción: los sistemas cuyo objetivo es la rentabilidad económica y aquellos cuya función es la satisfacción de las necesidades y la reproducción de la unidad de producción-consumo. En general, se ha encontrado que los sistemas orientados a la obtención de ganancia son más eficientes desde el punto de vista del flujo monetario, en tanto que los orientados a la reproducción de la unidad de producción-consumo son más eficientes energéticamente, dado que provocan menos transformación en los ecosistemas que los sistemas industrializados (Toledo, 1992).

Lo anterior se ha utilizado para sostener que los sistemas de producción cuyo objetivo es la reproducción de la unidad de producción-consumo son más sostenibles, dado que por sus limitaciones económicas y su propia racionalidad generan en los agroecosistemas procesos de reciclaje que les permite hacer mejor uso de la biomasa en sus sistemas de producción. No obstante, los avances empíricos que existen para corroborar esta hipótesis son muy escasos, pese a que en los últimos treinta años se ha reconocido la necesidad de los análisis de energía por su importante papel en la evaluación de la sostenibilidad de los sistemas agrícolas. En este sentido, Mora, Ramírez y Quirós (2007) han referenciado estudios de Pimentel, Beradi y Fast (1983), Zentner, Stumborg y Campbell (1989), Risoud y Chopined (1999), realizados para cuantificar el consumo de energía en la zona templada, y de Uhl y Murphy (1981), Hall y Hall (1993) en la zona tropical.

En Colombia, Flores, Sarandón y Lermanó (2007) destacan la importancia de las prácticas que contribuyen con la sostenibilidad de los sistemas agrarios. Al respecto afirman que los sistemas agroecológicos rescatan prácticas de la agricultura tradicional desde hace miles de años. Sin embargo, debido a procesos históricos y sociales dichas prácticas se han venido perdiendo y se ha adoptado un modo de producción menos amigable con la naturaleza, en la búsqueda de altos rendimientos productivos, pero con incorporación de insumos externos de alto consumo energético, y muy costosos.

Es evidente que la mayor sustentabilidad de los agroecosistemas, implica la disminución en el uso de insumos externos, especialmente los de síntesis química, al favorecer los procesos al interior de los mismos. Así, por ejemplo, el aumento de la biodiversidad, como estrategia para la regulación de las poblaciones de plagas y patógenos y el reemplazo de fertilizantes de síntesis química, muy costosos energéticamente, por abonos orgánicos y prácticas que estimulen la fijación biológica del Nitrógeno, se convierten en estrategias adecuadas para incrementar la estabilidad del sistema y, por lo tanto, disminuir el subsidio energético (Flores, Sarandón y Lermanó, 2007).

Alemán y Brito (2002) afirman que, como consecuencia del uso de insumos de alto contenido energético, ha disminuido la eficiencia energética; por ejemplo, el análisis histórico de la producción de maíz en Estados Unidos, entre 1945 y 1970, muestra que la energía empleada en este cultivo aumentó proporcionalmente con el incremento de los rendimientos; sin embargo, desde 1970 los incrementos de la demanda de energía no se traducen en ganancias similares en los rendimientos, 
lo cual significa que la producción agrícola se sostiene por el incremento de los insumos energéticos externos.

Adicionalmente, al contrastar un sistema de producción utilizando abonos orgánicos e insumos biológicos dio como resultado una alta eficiencia energética, frente al sistema tradicional mecanizado de altos insumos. En este sentido, la utilización de los residuos orgánicos derivados de las actividades de producción campesina que se reincorporen al sistema finca pueden constituirse en una fuente sustitutiva de insumos externos, disminuir las entradas de energía exógena, la contaminación de los cuerpos de agua y los costos.

En términos empíricos, varios estudios han permitido calcular la cantidad de energía empleada en diversos procesos productivos (Helsel, 1992; Fluck, 1995), cálculos que han permitido estimar coeficientes de conversión de la cantidad de insumos y mano de obra invertidos en un proceso productivo en términos de medidas energéticas. El propósito de dichos estudios ha sido analizar la energía que fluye en el sistema y establecer el respectivo balance energético entre entradas y salidas con diferentes medidas.

Al respecto, es importante destacar tres conceptos relacionados con las formas más conocidas de la medición de la energía en los sistemas agrícolas: a) eficiencia energética, que es la razón entre las unidades energéticas producidas y la energía invertida en los insumos; b) productividad energética, que relaciona la cantidad de producto obtenido por la energía invertida en el proceso de producción (Fluck \& Baird, 1980, Fluck, 1995) y c) rentabilidad energética, que es la relación entre ingresos generados y las unidades de energía invertida (Szott, 1998). Estas formas constituyen métodos complementarios a los análisis costobeneficio de diferentes alternativas o modelos productivos, y de esta manera se obtiene otra visión de la eficiencia de los sistemas de producción, que va más allá de lo estrictamente financiero.

Autores como Romero et al. (2002) destacan las ventajas del reciclaje de nutrientes en los sistemas productivos, planteando que la agricultura ecológica logra un mayor reciclaje de los nutrientes en la finca y disminuye la necesidad de fuentes externas de nutrientes. Así mismo, los sistemas agroforestales, extraen nutrientes desde diferentes profundidades y los incorporan al suelo, estableciendo un ciclo de nutrientes más estable, que los cultivos intensivos.

En los últimos años, se ha incrementado el interés y la necesidad de estudiar los flujos de energía involucrada en los procesos productivos. En este sentido, para hacer una valoración desde la perspectiva energética, se busca evaluar toda la energía involucrada en un producto, a partir de la energía utilizada en los procesos de transformación (Odum, 1986 y Odum, 1996 citados por Quintero, Hurtado \& Arango, 2009). Se trata de estimar el efecto que tiene la reutilización y reciclaje de residuos de producción y domésticos como fuente de energía y determinar los efectos financieros y económicos del manejo de estos en la finca, como sustitución de insumos de mayor costo. Con base en este análisis se podrá hacer una comparación entre los sistemas representativos y determinar cuál es más eficiente en términos energéticos y económicos.

En consecuencia, y en el contexto del proyecto: "Flujo de masas y energía en fincas campesinas de la zona cafetera: vínculos entre la racionalidad campesina y el reciclaje de materiales y energía", se realizó un trabajo de investigación con el objetivo de estimar la productividad energética y financiera en fincas de la zona cafetera del departamento 
de Caldas. Para obtener la información, se seleccionaron tres fincas, una de cada clúster resultante del proyecto mencionado anteriormente, en los municipios de Filadelfia y Manizales. La información se obtuvo mediante entrevista semiestructurada realizada con los miembros del hogar de cada finca. Para realizar los cálculos de productividad monetaria y energética, en cada finca, se seleccionó el sistema más representativo. Se busca, en últimas, determinar y comparar la eficiencia energética y financiera del componente principal en cada finca seleccionada.

\section{MATERIALES Y MÉTODOS}

\section{Localización}

El estudio se realizó en la zona centro sur y alto occidente del departamento de Caldas, en los municipios de Manizales y Filadelfia. El municipio de Manizales está localizado entre los $5^{\circ} 4^{\prime} \mathrm{N}-75^{\circ} 31^{\prime} \mathrm{O}$; a una altura de $1780 \mathrm{msnm}$, presenta una temperatura promedio de $21^{\circ} \mathrm{C}$ y una precipitación anual de $2888 \mathrm{~mm}$. Entre tanto, el municipio de Filadelfia está localizado entre $5^{\circ} 18^{\prime} \mathrm{N}-75^{\circ} 34^{\prime} \mathrm{O}$; a una altura de 1550 msnm, con una temperatura de $20^{\circ} \mathrm{C}$ y una precipitación anual de 2326 $\mathrm{mm}$.

Figura 1. Mapa zona de estudio.

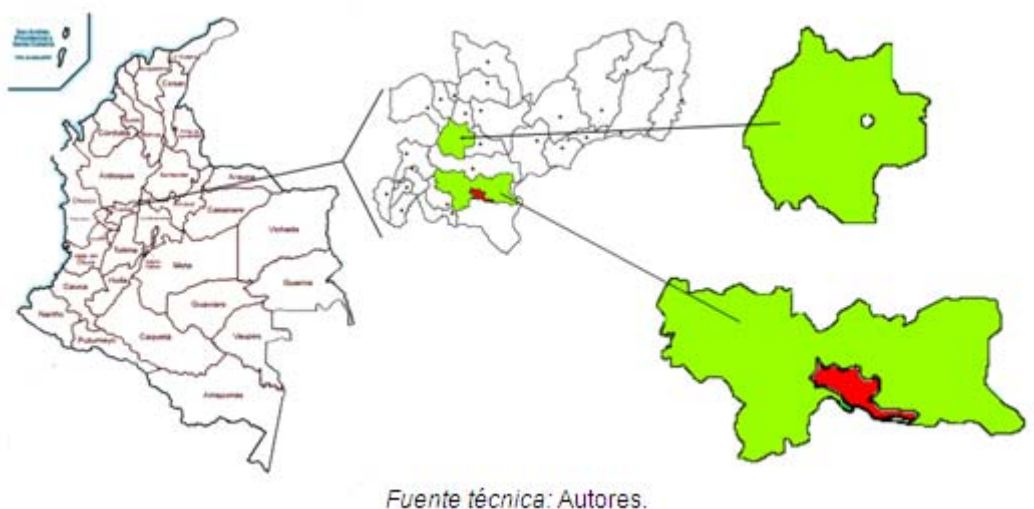

Técnicas e instrumentos

Para esta investigación se realizaron entrevistas semiestructuradas a tres familias de productores cafeteros en los municipios mencionados, con el fin de levantar información cualitativa y cuantitativa sobre sus actividades productivas.

Para la recolección de la información se diseñó un cuestionario, en el cual se registró la siguiente información: nombre de la finca, localización, composición familiar, composición de los sistemas productivos, prácticas agrícolas y pecuarias, insumos utilizados, cantidad de materiales residuales y su utilización, cantidad, precio y frecuencia de compra de insumos externos, costo del jornal, tiempo de dedicación de la mano de obra familiar o contratada, cantidad de producto por año en cada componente, y precio del producto. Dicha información se complementó con observaciones de campo. 
El análisis de la productividad energética y financiera del sistema productivo seleccionado en las tres fincas cafeteras, se realizó con base en la información recolectada. En primer lugar, se construyeron modelos analógicos para cada finca, con las entradas y las salidas del sistema café, componente principal. En segundo lugar, se estimó la productividad financiera y energética respectiva. Con base en estos resultados, se establecieron las comparaciones Productividad financiera vs. Productividad energética del componente principal del sistema de producción.

Tabla 1. Equivalencias energéticas de insumos y mano de obra.

\begin{tabular}{|c|c|c|}
\hline TIPO DE MATERIAL & UNIDAD & MJ/UNIDAD \\
\hline Fertilizante químico & $\mathrm{kg}$ & 10,8 \\
\hline Trabajo humano & dia/hombre & 7,0 \\
\hline Gasolina & litro & 34,78 \\
\hline
\end{tabular}

Fuente: Leach (1981).

Para calcular la productividad de la energía en los sistemas analizados, se estimó el uso de mano de obra ${ }^{6}$ e insumos que representan el gasto energético en las diferentes labores; para ello se utilizaron las equivalencias energéticas reportadas por Leach (1981) (Tabla 1). Además, se realizó un análisis de relación costo-beneficio (RCB), indicador que relaciona los valores monetarios de los ingresos y los correspondientes egresos, referidos a un año de actividad. Como se utiliza el mismo período para los tres casos, este indicador permite comparar la productividad financiera de cada una de las unidades productivas analizadas.

\section{RESULTADOS}

Los sistemas productivos correspondientes a las tres fincas cafeteras localizadas en los municipios de Filadelfia y Manizales (Caldas), tienen como común denominador la producción de café, componente principal en las unidades de producción agrícolas, y en menor proporción componentes pecuarios y otros cultivos de pequeña escala. Además, estas unidades se diferencian por la composición y el ciclo de vida de la familia.

La Finca 1, 'La Alejandra', corresponde a una unidad productiva localizada en el municipio de Filadelfia, con 10 hectáreas de superficie, un sistema productivo diversificado con cultivos de café, frutales, caña panelera, hortalizas y un área de cinco hectáreas de pasturas con capacidad para sostener 20 novillos; además cuenta con aves y peces, y área de conservación en bosque (Figura 2).

El propietario de la finca tiene experiencia y especial cuidado en la utilización de residuos orgánicos. Con ellos produce caldo "supermagro": una mezcla casera de leche, lixiviado de lombricultivos, sulfatos y estiércol de res. Otra de de las prácticas que realiza es la preparación del compost para el cultivo de café, lo cual le evita la compra de fertilizantes químicos. En su opinión, la no aplicación de insecticidas y fungicidas, favorece la existencia de insectos y arañas benéficos.

En la producción de café, la mano de obra utilizada es familiar. Laboran la madre, el padre y la hija mayor. La familia tiene un potencial ${ }^{7}$ de 416 
jornales por año. La mano de obra externa solamente se aplica a la caña panelera.

En cuanto a la composición familiar, además de los tres adultos, hay tres niños que estudian en la escuela de la vereda, y eventualmente colaboran en las labores agrícolas, lo cual favorece el arraigo de esta familia a su terruño y al desarrollo de prácticas productivas orientadas a la sostenibilidad de los agroecosistemas.

Figura 2. Diagrama de flujo finca 'La Alejandra'. Municipio de Filadelfia (Caldas).

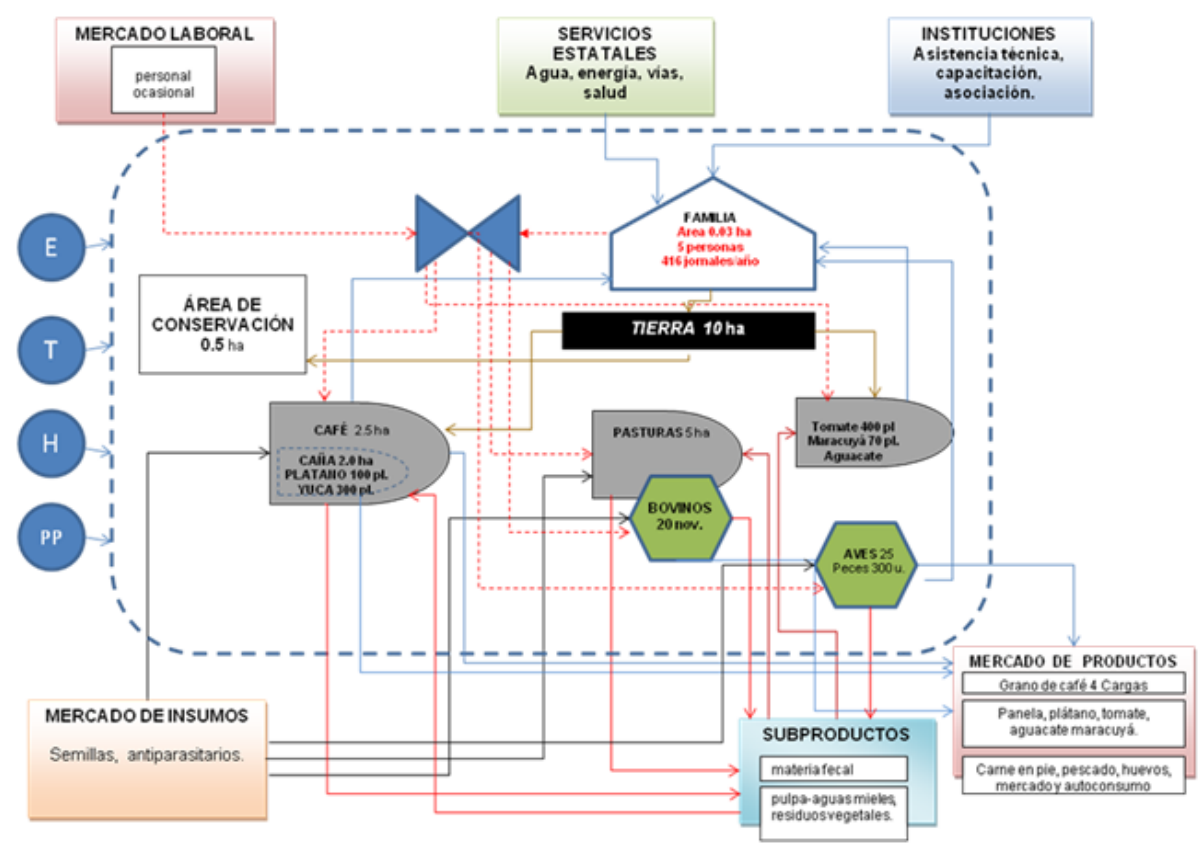

La Finca 2, 'Casagrande', está localizada en la vereda Pueblo Hondo, aproximadamente a 30 minutos de Manizales, capital del departamento de Caldas. Tiene un área de 8 hectáreas, distribuida en diversos cultivos: café, banano, plátano, hortalizas, flores e instalaciones de producción de cerdos, conejos, peces y aves, además de un área en bosque, como se muestra en la Figura 3.

En cuanto a la estructura familiar, aunque tiene el mismo número de miembros que la Finca 1, su potencial de trabajo es mayor. Está integrada por cuatro adultos: dos mujeres y dos hombres, y dos niños en edad escolar. El potencial de trabajo de la familia está representado en unos 850 jornales por año. Las decisiones de manejo de la finca se toman por consenso, en cabeza de la madre, que cuenta con gran capacidad de liderazgo familiar y comunitario, el padre y el hijo mayor quien tiene formación en técnicas agropecuarias y una gran convicción del valor que representa trabajar la tierra y vivir en y de el campo.

La finca presenta una gran diversidad agrícola y pecuaria, lo que le permite a la familia la utilización de los residuos de cada subsistema, sobre todo el biodigestor y el compost generado por el componente pecuario de cerdos y conejos. Además, el componente agrícola es diversificado, con café, plátano, banano, yuca, frutales y huerta.

Figura 3. Diagrama de flujo finca 'Casagrande'. Vereda Pueblo Hondo (Manizales, Caldas). 


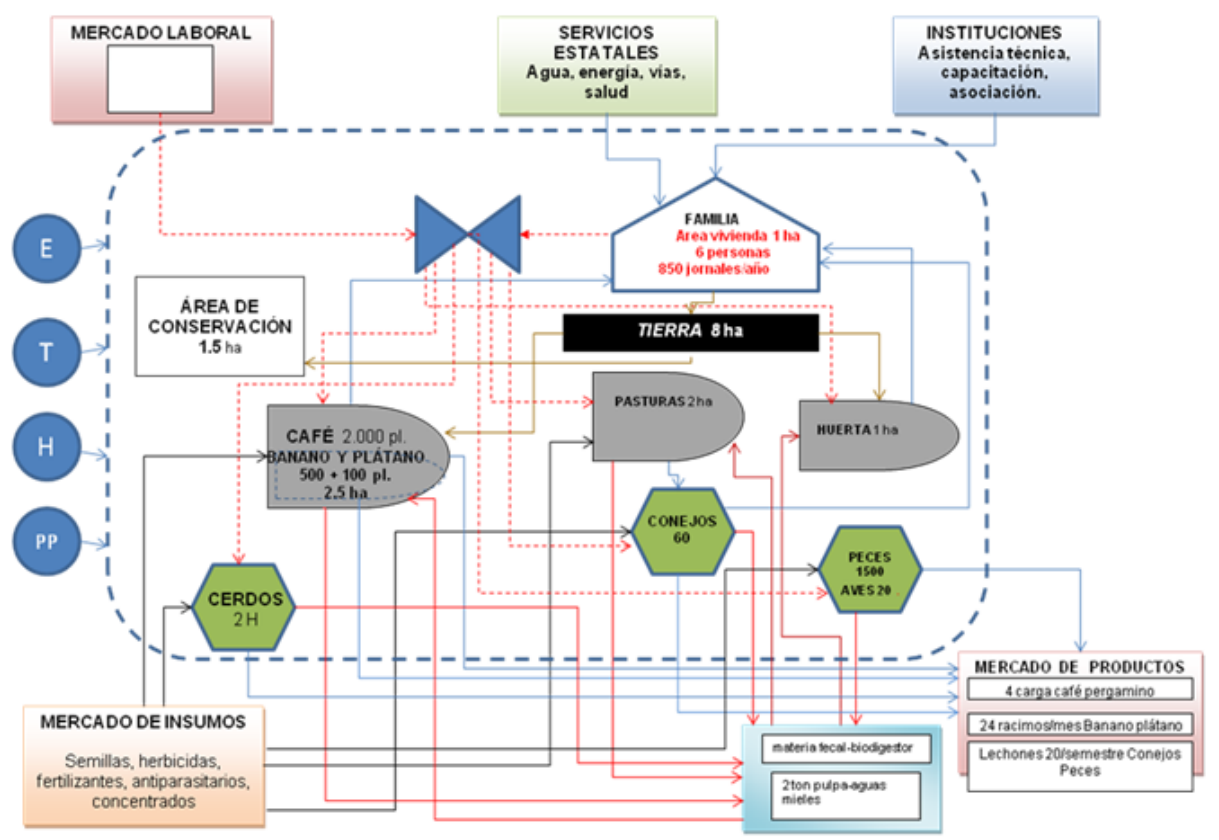

La Finca 3, 'Villa Claudia', con área de 5 hectáreas, está localizada en la vereda Santa Clara, municipio de Manizales. Se caracteriza por ser un sistema convencional centrado en la producción de café, con utilización de fertilización química, insumos externos para control de plagas y enfermedades, mano de obra contratada y mínima utilización de material orgánico derivado de los residuos del beneficio del café, aunque se hace un manejo mínimo de estos residuos mediante compostaje. El hogar está compuesto por dos adultos mayores, y dos hijos adultos, que laboran independientemente en la ciudad de Manizales (Figura 4).

Figura 4. Diagrama de flujo finca 'Villa Claudia'. Vereda Santa Clara (Manizales, Caldas).

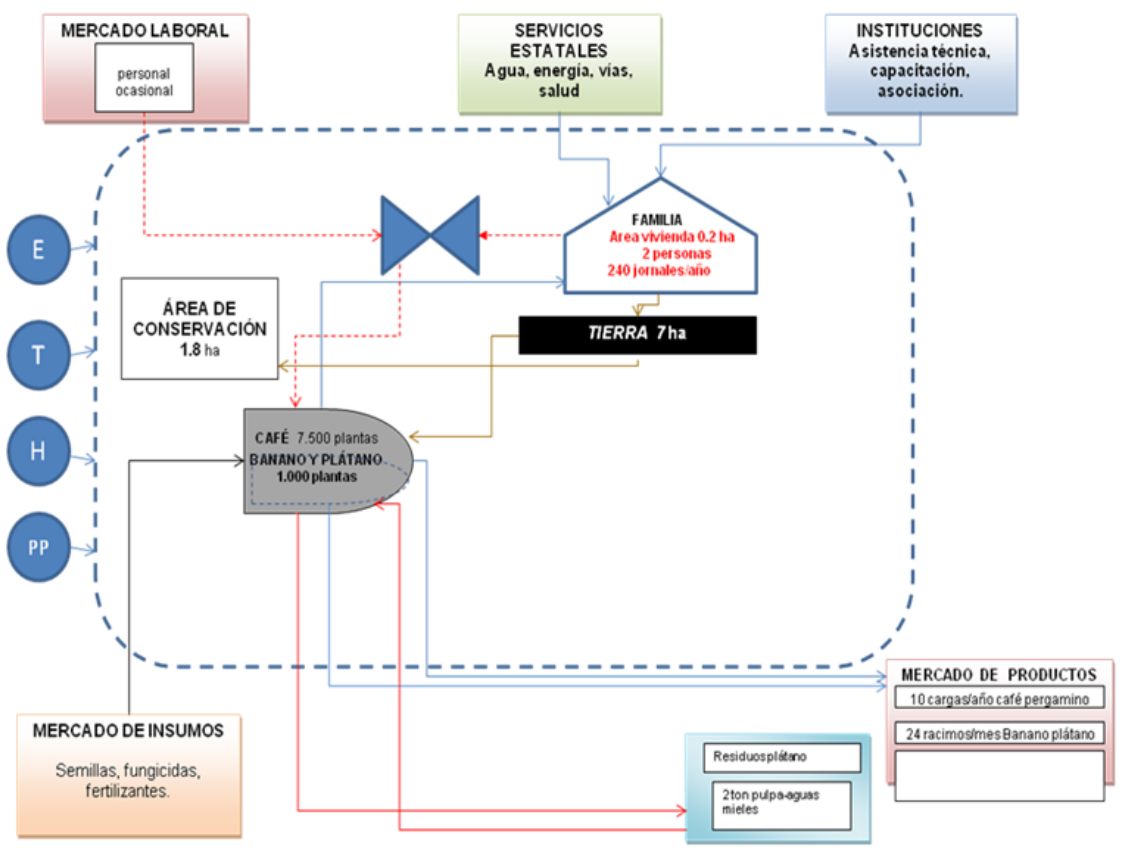


En la Tabla 2 se muestran los resultados financieros y energéticos de cada uno de los casos representativos, con datos de costos de insumos y producción por año.

Tabla 2. Rentabilidad y productividad financiera y energetica en fincas cafeteras de Caldas (Colombia).

\begin{tabular}{|c|c|c|c|c|c|c|c|c|c|}
\hline FINCA & No. & MUNICIPIO & $\begin{array}{c}\text { EGRESOS } \\
\text { (C) }\end{array}$ & $\begin{array}{c}\text { INGRESOS }^{* \star * x} \\
\text { (B) }\end{array}$ & $\mathrm{RBC}^{*}$ & ENTRADAS & SALIDAS & $\begin{array}{l}\text { PRODUC TIVIDAD } \\
\text { ENERGÉTICA }\end{array}$ & $\begin{array}{c}\text { RENTABILIDAD } \\
\text { ENERGÉTICA }\end{array}$ \\
\hline & & & (S/año) & (S/año) & $B / C$ & $\mathrm{MJ}^{* \star} / a$ año & $\mathrm{kg}$ cafélaño & $\mathrm{kg}$ café/MJ & $\$ / M J$ \\
\hline $\begin{array}{c}\text { La } \\
\text { Alejandra }\end{array}$ & 1 & Filadelfia & 1'109.000 & 3'235.864 & 2,92 & 329,00 & 500 & 1,52 & 9.835 \\
\hline Casagrande & 2 & Manizales & 1'574.118 & $3^{\prime} 235.864$ & 2,38 & $4.083,7$ & 500 & 0,12 & 792 \\
\hline $\begin{array}{l}\text { Villa } \\
\text { Claudia }\end{array}$ & 3 & Manizales & $6 ' 371765$ & $8^{\prime} 089660$ & 147 & 18470 & 1250 & 0.07 & 438 \\
\hline
\end{tabular}

ANÁLISIS

DE

LOS

RESULTADOS

La eficiencia financiera se evaluó mediante el cálculo de la relación beneficio-costo (RBC), indicador que relaciona los valores monetarios de los ingresos y los correspondientes egresos, referidos a un año de actividad. Al utilizar el mismo período para los tres casos, el indicador permite comparar la eficiencia financiera del componente principal, de cada una de las unidades productivas analizadas.

El análisis de los resultados desde el punto de vista costo-beneficio, tomando los precios del café de julio de 2010, muestra un mejor desempeño en la Finca 1 localizada en el municipio de Filadelfia (Caldas) con una RBC de 2,92, seguida de la Finca 2 con 2,38, y finalmente la Finca 3 con un valor de 1,47. De acuerdo con el análisis realizado, las diferencias de la $\mathrm{RBC}$ radican principalmente en el manejo de materiales orgánicos reciclados en la finca y en la preparación de abonos orgánicos. Así mismo, la capacidad para desarrollar los procesos de reciclaje en la Finca 1 se expresa en una mayor RBC como consecuencia de la no utilización de fertilizantes químicos.

En la Finca 2, que le sigue en productividad financiera, aunque se utilizan fertilizantes químicos, también se acostumbran algunas prácticas de reciclaje de materiales y sus componentes pecuarios permiten desarrollar procesos de utilización cada vez más eficientes. La Finca 3, de manejo más convencional, depende de insumos externos, principalmente fertilizantes. Es menos diversificada y carece de componentes pecuarios. Su RBC es la menor.

Desde el punto de vista de la productividad energética, las entradas de energía se valoran en Megajulios (MJ). Se estableció la relación de cantidad por unidad de producto por cada MJ utilizado en el proceso de producción, obteniéndose una mayor productividad energética en la Finca 1, con un valor de 1,52 kilogramos de café por MJ, seguida de la Finca 2 con una productividad energética de $0,12 \mathrm{~kg}$ de café por MJ, y finalmente la Finca 3 con $0,07 \mathrm{~kg} / \mathrm{MJ}$.

La relación de productividad entre las tres fincas es 22:13:1; es decir, la Finca 1 produce 22 veces más café que la Finca 3 por cada unidad energética (MJ) utilizada. En términos de rentabilidad energética, ingresos monetarios generados por cada unidad de energía utilizada, claramente se observa que la Finca 1 tiene la mayor rentabilidad con un valor de $\$ 9.835$ por cada MJ utilizado.

Si bien, la estimación de la productividad energética y financiera y la comparación entre los casos estudiados, permiten concluir que la Finca 
1 presenta la mejor RBC y mejor eficiencia energética, también permite señalar que esta, por su mayor diversidad, mejor aprovechamiento de los residuos y estructura familiar, es una unidad más resiliente y resistente a los disturbios del entorno natural y social, lo cual la hace más sostenible desde la óptica social, económica y ecosistémica.

\section{CONCLUSIONES}

Del estudio realizado se pueden derivar las siguientes conclusiones:

El uso de los materiales residuales de las fincas cafeteras adecuadamente procesados, puede sustituir la fertilización con productos derivados de la síntesis industrial y la utilización mayoritaria de mano de obra, familiar principalmente, producen mejor efecto financiero y uso más eficiente de la energía, corroborando los hallazgos de Lockeretz, Shearer, Kohl y Klepper (1984), Karlen, Duffy y Colvin (1995), Languë y Khelifi (2001), que demuestran que se pueden lograr importantes ahorros de energía al sustituir insumos convencionales altos en energía indirecta, por insumos orgánicos o prácticas culturales.

Existe relación directa entre productividad energética y financiera, es decir que, al menos, para sistemas de producción cuyo componente principal es el café, tengan un nivel importante de diversificación y utilicen mano de obra familiar, la productividad energética y financiera siguen la misma tendencia.

Con base en los resultados, se puede rebatir el argumento según el cual los sistemas de producción industrializados y más especializados son más eficientes financieramente que los tradicionales y menos diversificados. Lo anterior indica que el reciclaje de materiales y energía en las fincas, antes que afectar la rentabilidad financiera la favorece. Con ello, se concluye que dichos sistemas son más sustentables en términos económicos, sociales y ecosistémicos.

La sustentabilidad de la producción en las fincas campesinas no solo debe considerar los flujos monetarios y la rentabilidad, sino también los flujos de biomasa y energía. De acuerdo con los cálculos realizados, las fincas en las cuales se reutilizan los desechos orgánicos son más productivas energética y financieramente. El argumento sobre el cual se sustenta lo anterior, deriva del hecho de que si bien los agricultores le asignan mayor importancia a los flujos financieros en la toma de decisiones en sus fincas, sin considerar los flujos de materia y energía que circulan en sus unidades productivas, son estos últimos los que garantizan la sustentabilidad de sus sistemas de producción y el bienestar de sus hogares.

Del análisis realizado se puede afirmar que si bien la lógica de la producción campesina está orientada por una racionalidad ecológica con arreglo a valores, la lógica del sistema de mercado hace que sus decisiones estén guiadas más por cuestiones económicas y financieras. En principio, los productores preparan abonos orgánicos porque les representa un ahorro de dinero al disminuir la compra de fertilizantes de síntesis química. Lo que, en primera instancia, induciría a pensar que su lógica es más económica que ecológica. Pero no hay tal. La forma como se toman las decisiones en las unidades campesinas es más compleja, incluye muchos factores que escapan a los análisis convencionales realizados. 


\section{BIBLIOGRAFÍA}

- Alemán, R., y Brito, J. (2007). Balance Energético en dos sistemas de producción de maíz. Centro de Investigaciones Agropecuarias (CIAP). Universidad Central "Marta Abreu". Cuba.

- Calderón C., P. A. (2009). Planificación Participativa de Fincas con Modelos Agrosilvopastoriles: Alternativa para la Productividad Agropecuaria y la Sustentabilidad en el Departamento de Caldas. Tesis, MSc. Sistemas de Producción Agropecuaria, Universidad de Caldas Manizales, Colombia.

- Flores, C., Sarandón, S., y Lermanó, M. (2007). Eficiencia Energética en Sistemas hortícolas familiares del Partido de La Plata, Buenos Aires, Argentina. Resumos do II Congresso Brasileiro de Agroecologia.

- Fluck, R. C. (1995). The hidden input. Southern Regional Workshop Evaluating Sustainability. Florida, US: University of Florida. pp. 31-43.

- Fluck, R., y Baird, C. (1980). Agricultural energetic. Universisty of Florida, USA: Avi Publishing.

- Hall, C. A. S, y Hall. M. P. H. (1993). The efficiency of land and energy use in tropical economies and agriculture. Agricultural, Ecosystems and Environment, 46, 1-30.

- Helsel, Z. R. (1992). Energy and alternatives for fertilizer and pesticide use. En Fluck, R. C. (ed.), Energy in farm production (pp. 177-201.). New York: Elsevier.

- Karlen, D. L., Duffy, M., y Colvin, T. (1995). Nutrient, labor, energy and economic evaluations of two farming systems in lowa. Journal of Production Agriculture, 8(4), 540-546.

- Languë, C., y Khelifi, M. (2001). Energy use and time requirements for different weedings strategies in grain corn. Canadian Biosystems Engineering, 43, 2.13-2.21.

- Leach, G. (1981). Energía y producción de alimentos. Madrid, España: Secretaría General Técnica del Ministerio de Agricultura y Pesca.

- Lockeretz, W., Shearer, G., Kohl, D., y Klepper, R. (1984). Comparison of organic and conventional farming in the corn belt. En Organic farming: current technology and its role in a sustainable agriculture (pp. 37-48). Madison, WI: American Society of Agronomy.

- Mizgajski, A. (1988). Historical changes of energy input into agroecosystems and their landscape ecological consequences, the Lingen/Emsland County (West Germany) Case Study. Agriculture, Ecosystems and Environment, 20, 245-258.

- Mora, J. R., Ramírez, C., y Quirós, O. (2007). Mano de obra, análisis beneficio-costo y productividad de la energía en la caficultura campesina de Puriscal, Costa Rica. Cuadernos de Administración, 20(033), 79-101.

- Pimentel, L. D., Beradi, G., y Fast, S. (1983). Energy efficiency of farming systems: organic and conventional agriculture. Agriculture, Ecosystems and Environment, 9, 359-372.

- Quintero, L., Hurtado, L., y Arango, S. (2009). Evaluación de sostenibilidad en pequeñas unidades de producción agrícola mediante dinámica de sistemas. En U. I. Santander, Latinoamérica Una comunidad que aprende dinámicas de sistemas y con dinámicas de sistemas ( $\mathrm{pp}$. 705-714).

- Risoud, B., y Chopinet, B. (1999). Efficacité énergétique et diversité des systems de production agricole, Applications a des exploitation bourguignonnes. Ingenieries, 20, 17-25.

- Romero, J. C., et al. (2002). Producción Agroecológica. Coordinadora Ecuatoriana de Agroecología -CEA-. Quito, Ecuador: Consorcio Camaren. 
- Szott, L. (1998). Energía en la agricultura. Guía del curso de Agricultura Tropical Sostenible. Programa de Doctorado SPATS, UCR. Costa Rica. Multicopiado.

- Toledo, V. M. (1992). La racionalidad ecológica de la producción campesina. En Sevilla Guzmán, E., y González de Molina, M. (eds.), Ecología, campesinado e historia (pp. 197- 218). Madrid: La Piqueta.

- Uhl, C., y Murphy, P. (1981). A comparison of productivities and energy values between slash and burn agriculture and secondary succession in the upper Rio Negro region on the Amazon Basin. Agroecosystems, 7, 63-83.

- Zentner, R., Stumborg, M., y Campbell, C. (1989). Effect of crops rotations and fertilization on energy balance in typical production systems on the Canadian prairies. Agriculture, Ecosystems and Environment, 25, 217232.

1. Doctor en Sociología del Medio Ambiente. Profesor Universidad de Caldas. E-mail: isaias.tobasura@ucaldas.edu.co

2. Magíster en Economía. Profesor Universidad de Caldas. E-mail: fred.moreno@ucaldas.edu.co

3. I.A. Estudiante Maestría en Sociedades Rurales. E-mail: sandra.aya@ucaldas.edu.co

4. Doctor en Sistemas de Producción Agrícola. Profesor Asociado, Universidad del Tolima. E-mail: jrmora@ut.edu.co

5. Proyecto realizado en municipios de los departamentos de Tolima y Caldas, durante los años 2009 y 2010, por la Universidad del Tolima y la Universidad de Caldas, financiado por la Red Alma Máter.

6. Se usa aquí el concepto de porción diaria del contenido de energía por alimento consumido, que según reportes de la literatura oscila entre 6,4 y $8,0 \mathrm{MJ}$, lo cual difiere del gasto de energía muscular que es la verdadera energía termodinámica que oscila entre 0,4 y $0,7 \mathrm{MJ} /$ día (Fluck \& Baird, 1980; Fluck, 1995; Mizgajski, 1988).

7. El potencial de trabajo se calcula a partir del número de horas que cada miembro de la familia dedica al trabajo del componente principal. 